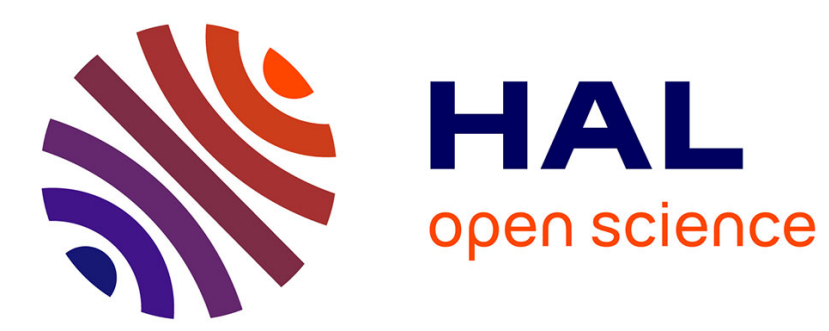

\title{
Du local sans idée aux idées territoriales en action
} Emmanuel Négrier

\section{To cite this version:}

Emmanuel Négrier. Du local sans idée aux idées territoriales en action . 2017. hal-01439645

\section{HAL Id: hal-01439645 \\ https://hal.science/hal-01439645}

Preprint submitted on 18 Jan 2017

HAL is a multi-disciplinary open access archive for the deposit and dissemination of scientific research documents, whether they are published or not. The documents may come from teaching and research institutions in France or abroad, or from public or private research centers.
L'archive ouverte pluridisciplinaire HAL, est destinée au dépôt et à la diffusion de documents scientifiques de niveau recherche, publiés ou non, émanant des établissements d'enseignement et de recherche français ou étrangers, des laboratoires publics ou privés. 


\title{
« Du local sans idée aux idées territoriales en action»
}

\author{
proposition de contribution à l'ouvrage en l'honneur de Bruno Jobert
}

Emmanuel Négrier

Le rôle des idées dans les politiques publiques a surtout fait l'objet de débats et développement dans l'analyse de politiques sectorielles. Le niveau national de leurs agenda et mise en œuvre a été privilégié comme angle d'attaque et comme base de comparaison. Les politiques transversales ont été moins souvent mobilisées, même si elles ont démontré que l'articulation entre idées et action publique y était également pertinente, tout en soulevant les débats mentionnés en introduction.

Les politiques territoriales, qu'il s'agisse de la territorialisation des politiques publiques ou de la mise en œuvre de politiques visant expressément le champ d'intervention et de pouvoir territoriaux, n'ont pas bénéficié d'un éclairage équivalent. Pourtant, on peut penser que les mérites analytiques de cette articulation y sont de plus en plus sensibles.

Les questions territoriales ont engendré des politiques soutenues par un référentiel relativement cohérent autour de la notion de modernisation. Elles ont permis une lecture largement dépolitisée des enjeux du pouvoir local, dominés qu'ils étaient par les notions de marchandage et de négociation à la marge. Ces mêmes questions se transforment aujourd'hui. Elles tendent à s'orienter vers des choix marqués en apparence par le défaut d'idéologie, en se revendiquant d'objectifs de rationalisation financière ou en s'investissant sur le cadre constituant (décentralisation, incitation à l'émergence de nouvelles structures) plus que sur la substance de l'action publique. Une première hypothèse pourrait laisser penser à un système de vases communicants, la dépolitisation des enjeux nationaux allant de pair avec la repolitisation des questions territoriales. Simpliste, elle traduit pourtant à la fois l'augmentation de la part relative des pouvoirs locaux dans la mise en œuvre des ressources de l'action publique, et l'emprise que cherchent à exercer sur elle les producteurs d'idées.

Une vision un peu plus élaborée montre qu'au-delà de ce jeu à somme nulle, c'est le produit de la combinaison entre échelles qui est au cœur des transformations. On peut ainsi montrer que ces processus conduisent à de nouvelles formes de politisation inter-niveaux, et à un changement du sens des relations entre niveaux, depuis le modèle de l'ajustement à la marge dans un cadre relativement négocié et stable jusqu'à un problème permanent de mise en cohérence de logiques fondées sur des jeux politiques instables.

\section{$1^{\circ}$ Les transformations politiques de la question territoriale}

L'évolution de l'approche territoriale du politique et des politiques publiques est celle des controverses qui ont progressivement déplacé les axes de recherche, les lignes de débat et les sources d'inspiration scientifique. Pour comprendre cette évolution, et la mettre en perspective de la question démocratique, on peut la présenter sous la 
forme d'une transformation de la dialectique dominante qui l'affecte, dans les rapports entre centre et périphérie. En effet, si la question territoriale ne cesse jamais d'être simultanément centrale, elle a connu de profondes mutations dans la manière d'articuler les différents niveaux de perception et de spatialisation. Dans cette évolution des représentations, l'analyse des politiques publiques croise en permanence une interrogation sur le politique.

\subsection{Le local sans idée}

Les controverses scientifiques sur les relations entre territoire et politique ont d'abord été alimentées par une dialectique verticale. Les paradigmes en usage dans les années 1970 et 1980 montrent que la critique scientifique était à la recherche de points d'équilibre ou de tension entre centre et périphérie. La régulation croisée aura été I'un des instruments les plus heuristiques (Grémion 1976) pour qualifier cette première période d'une critique de la territorialité. Elle s'attaquait elle-même à la domination d'un discours normatif, souvent d'ordre juridique, qui produisait une fausse naturalité d'un territoire réduit à l'expression de la puissance publique d'État (Alliès 1980). Ce faisant, elle énonçait une certaine contingence du territoire à l'égard du politique. Elle se déduisait de l'analyse des interactions concrètes entre mondes que le droit, lui, séparait pour mieux les aligner l'un sur l'autre. La dépendance, idéologique et décisionnelle, dans laquelle se trouvaient les pouvoirs locaux, pouvait dès lors fonder un « modèle hétéronome », par lequel ces derniers étaient conduits à appliquer, plus qu'à engendrer eux-mêmes, les normes pénétrant le territoire depuis les centres décisionnels de l'appareil d'État. Les expérimentations de certaines grandes villes, où des projets pouvaient conduire à des liens inédits entre élus, experts et bureaucraties locales (Jobert et Sellier 1977: 215), n'étaient que des exceptions à la règle d'un local sans idées.

Cette vision d'un local sans idée est largement partagée. La géographie, la sociologie et l'économie, qui s'intéressaient à ces enjeux territoriaux, utilisaient des démarches voisines. Les recherches sur la planification urbaine (Lojkine 1972), sur la ville (Castells 1974, Lefèbvre 1973), ou sur les rapports socio-économiques à l'échelle globale (Amin 1973) étaient orientées vers un dévoilement des fausses évidences du couple central/local, qu'elles soient d'essence normative (fausse égalité, Dulong 1978) ou localiste (fausse identité, Sfez 1977). C'est que les visions néo-marxistes ou systémiques, fondées sur les acquis de la sociologie des organisations, conduisaient elle-même à promouvoir un ordre.

Pour la science politique, la mise en évidence de la régulation croisée induisait l'existence d'une stabilisation des jeux à partir de laquelle pouvaient s'enclencher les petits et grands arrangements avec la règle. La négociation supposait elle-même un ordre «central», dont la cohérence ressortait presque intacte, voire dotée d'un supplément d'efficience. En termes de science politique, Albert Mabileau résumait bien l'équation d'un « local qui ne se laissait regarder qu'en creux du national ». Une telle tendance était également démontrable dans d'autres sciences sociales, avec lesquelles la science politique n'engageait d'ailleurs que peu de dialogue véritable, sauf exception. Cette dialectique verticale induisait l'emploi du singulier pour chacun des termes: le politique; le territoire. Le premier était lui-même scindé en deux 
acceptions : le politique (au masculin) pour exprimer le sujet de la régulation, et la politique (« politicienne » et territoriale) pour désigner l'objet de cette régulation.

Cette tendance à la stabilisation des jeux autour de la notion de régulation allait en France connaître une remise en question graduelle par la mise au jour d'exceptions à la règle, comme celle du pouvoir des grands élus urbains, disposant de ressources politiques trop importantes - et notamment par le cumul des mandats - pour être cantonnés au monde de la «petite politique » périphérique (Garraud 1983). Cette exception en rejoignait d'autres, fondées par exemple sur des caractéristiques, comme les cultures politiques (Lenclud 1986), propres à certains espaces (la Corse, le Languedoc, la Bretagne...). L'analyse des configurations urbaines et de leurs modes de différenciation à l'intérieur des cadres nationaux ouvrait sur un autre débat entre hétérogénéité socio-économique et intégration politique (Jobert 1977a). De telles différences « horizontales », montant en importance, ont complexifié la donne au point de rendre un peu artificiel le primat de la négociation verticale et publique. Les premiers mouvements de décentralisation, au début des années 1980, ont sonné le glas d'une telle dialectique verticale.

\subsection{Une dialectique horizontale}

Cette nouvelle phase a été considérablement une science politique plus sensible au dialogue avec d'autres sciences sociales (géographie, économie, sociologie), qui ont pris au sérieux la territorialité des enjeux politiques et sociaux. Elle a conduit à l'émergence d'une deuxième dialectique que l'on peut sommairement définir comme horizontale. Elle a contribué au développement de nouvelles notions comme celles de gouvernement urbain (Borraz 1998, Jouve et Lefèvre 1999), de gouvernance (John 2001, Leresche 2001), d'échange politique territorialisé (Négrier 1998), de relations public-privé (Le Galès 1997) ou encore de subsidiarité (Faure 1998). Elles ont, toutes, cherché à mettre en évidence l'influence de variables jusque-là restées dans l'ombre d'une appréhension verticale des enjeux d'action publique et des jeux de pouvoir.

Pour la sociologie du politique en particulier, on retrouve de telles perspectives au cœur du développement d'analyses localisées du politique (Briquet 1997, Sawicki 1997). Elles puisent leurs racines dans des travaux plus anciens, de type monographiques, qui s'intéressent à la construction locale du pouvoir (Lagroye 1972, par exemple). Elles croisent les innovations liées l'anthropologie politique, à la faveur du «retour » de l'ethnologie française sur le territoire national (Abélès 1989, Pourcher 1995). La démarche n'est pas tant, dans la veine d'Albert Mabileau, d'identifier le local autrement que comme une sorte de «ventriloque de l'Etat », en s'intéressant aux facteurs locaux de la politique nationale. Elle n'est pas davantage, dans la perspective naissante d'une socio-histoire du politique et de l'administration, de montrer ce que l'identité bureaucratique et territoriale française doit à la montée en puissance, par-le-bas, d'un groupe social et de routines institutionnelles (Dumons, Pollet, Saunier 1997). L'idée commune à l'ensemble de ces courants est que le regard fondé depuis le centre (des organisations partisanes, des bureaucraties et professions, des réglementations) était devenu largement insuffisant pour aborder un 
chantier de recherche sur des objets aussi centraux que l'espace, le pouvoir, la légitimité... le territoire.

Si la première dialectique tentait de compenser la force des relations verticales par une certaine horizontalité des pratiques (les relations préfet-notables, par exemple), la deuxième a tenté d'échapper au « localisme analytique » par d'autres dimensions verticales ou inter-locales. La compétition économique, le benchmarking territorial, l'européanisation ou la représentation des intérêts privés sont des variables qui naissent à la fin des années 1990 pour problématiser la territorialisation et la faire échapper à une vision romantique de l'autonomie locale. Cette deuxième critique s'est largement appuyée sur le cadre de la globalisation et sur l'émergence de politiques locales pour montrer tous les bénéfices, mais aussi toutes les limites de la territorialité des États en action.

Dans la construction de ces deux critiques successives, une différence fondamentale a trait aux travaux qui constituent l'environnement intellectuel de la pensée sur la territorialisation. La première s'appuie presque exclusivement sur une littérature critique française, et raisonne en contrepoint de formalisations juridiques également nationales. Si I'approche organisationnelle puisait naturellement ses racines dans des fondements anglo-saxons, elle restait fondamentalement française dans la structure de son débat conceptuel et empirique. La deuxième critique est par contre fortement informée des recherches internationales. Elle se réfère à un large spectre de travaux, du renouveau de l'analyse institutionnelle (Hall 1993, Hall \& Taylor 1996), aux réflexions naissantes en termes de gouvernance (Jessop 1997, Marks 1996), ou de régime urbain (Harding 1994). L'évolution de l'analyse régionale s'appuie sur des programmes de recherche comparatifs (Keating \& Loughlin 1996; Jeffery 1997) dans lesquels s'inscrivent désormais les travaux français (Le Galès \& Lequesne 1998, Négrier \& Jouve 1998). Les questions métropolitaines s'émancipent d'une stricte vision nationale pour se situer dans des débats (reform vs public choice, par exemple) qui sont parfois fort anciens dans le monde anglo-saxon (Woods 1958, Ostrom, Tiebout \& Warren 1961).

L'importation de modèles d'analyse ou de concepts (governance, urban regimes, new-regionalism ou new-institutionalism, par exemple) ne se fait pas sans débat au sein du champ scientifique français. La gouvernance urbaine en est l'un des plus clairs exemples (Jouve \& Lefèvre 1999; Lorrain 2000; Gaudin 2002 ; Le Galès 2002). Cette importation intègre cependant, de plus en plus, la diversité interne des positions abusivement considérées, parce qu'anglo-saxonnes, comme cohérentes entre elles. En outre, d'autres emprunts, plus continentaux, font en même temps leur apparition dans la science française du territoire. On peut ici mentionner la fortune qu'a connue la littérature italienne des districts industriels autour de Carlo Trigilia (1986) notamment (Ritaine 1989, Benko \& Lipietz 1992); ou de celle sur l'échange politique (Pizzorno 1977 ; Ceri 1981).

Cette importation a aussi permis de questionner l'hypothèse d'une spécificité radicale du cas français, et de l'intégrer de façon ordinaire dans la comparaison de certaines politiques territoriales, comme nous allons le voir. Dans ce même mouvement, la question territoriale aura cessé d'être celle de la pure mise en œuvre d'algorithmes centraux et antérieurement construits. Elle s'ouvre à des interrogations, empiriques 
et théoriques, portant sur le sens de l'action publique, et donc sur l'idéologie des politiques territoriales (Arnaud, Le Bart et Pasquier 2005).

\section{Un nouveau cycle de territorialisation}

Comment concilier l'horizontalité et la verticalité nécessaires des modes d'analyse, dans un contexte marqué par des transformations continues des politiques territoriales ? La décentralisation et l'européanisation des politiques publiques, de même que les changements d'échelle du gouvernement local sont les objets majeurs de cette interrogation. Les déplacements de centre de gravité de l'action publique imposent une double réflexion. La première concerne l'hypothèse d'une fin de cycle de I'action publique territoriale. La seconde a trait aux instruments d'analyse pour en rendre compte.

\subsection{Une inversion politique}

La notion de fin de cycle est souvent critiquée, en ce qu'elle fait précisément I'hypothèse de cycles eux-mêmes abusivement simplifiés et historiquement bordés. Elle doit donc être utilisée avec prudence, pour qualifier des inversions de tendance dans plusieurs secteurs d'un même domaine, inversions qui participent, par hypothèse, d'un même mouvement de fond. En matière de territorialisation, on peut s'appuyer sur plusieurs phénomènes simultanés, qui concernent les jeux d'échelle, la substance des relations entre acteurs et la nature des instruments propres à leur mise en œuvre.

Les changements d'échelle qui marquent la territorialisation d'aujourd'hui sont à la fois administratifs, politiques et spatiaux. La décentralisation a connu de nouvelles impulsions en France, et marque le déclin d'un certain jacobinisme territorial. Mais ce qui la caractérise est aussi la fin d'une croyance aux vertus intrinsèques de la proximité en termes de démocratisation, d'économie d'échelle, d'efficience. Les destinataires des nouvelles compétences n'ont pas été les derniers à incarner un scepticisme inédit, mal compensé par une contrepartie de type constitutionnel sur l'économie des transferts de charge. La fin du mythe décentralisateur, qui coïncide avec la progression de la décentralisation, s'accompagne de changements dans les relations entre échelles.

Quant aux échelles du gouvernement local, elles ont connu un véritable changement de régime. Après des résultats plus que limités dans les années 1990 - la loi Joxe Baylet de février 1992 n'ayant par exemple conduit qu'à la création de trois communautés de ville - la loi Chevènement, portant sur le même objet, a connu un succès considérable, avec la création, en six ans, de plus de 180 intercommunalités urbaines et de plus de 2300 communautés de communes, à un niveau démographique plus modeste. C'est l'absence d'intercommunalité qui est devenue l'exception, alors qu'elle était la règle à la fin du XXème siècle. Bien sûr, il convient d'observer toutes les limites de cette « révolution intercommunale », à commencer par la grande diversité entre situations que cache un tel mouvement. Mais elles ne 
sauraient contester la réalité d'une transformation profonde de l'économie politique des pratiques gouvernementales locales.

Les relations entre échelles suggèrent également quelques transformations conséquentes. Qu'en est-il de la relation des territoires à l'État dans un contexte de décentralisation? En Espagne, l'administration centrale, d'ailleurs tour à tour qualifiée $d^{\prime}$ " espagnole » ou de «castillane », apparaît porteuse d'intérêts chaque fois plus spécifiques, et reçus comme tels dans les communautés autonomes, terrains d'impulsion de politiques publiques de plus en plus ... centrales (Subirats \& Gallego 2002). En France, par un contraste apparent, la croyance résiduelle en I'État semble résister au transfert de ses propres attributions. La multiplication des baromètres d'opinion localisés confirme le fait qu'il continue de figurer comme porteur de politiques (RMI, formation) qu'il n'a pourtant plus dans ses attributions. Mais, au-delà de cette croyance, ce rapport entre échelles s'est considérablement transformé aujourd'hui. Les collectivités territoriales sont désormais en charge d'une proportion croissante de ce que l'on dénomme encore l'État providence, et qui se réfère aujourd'hui aux politiques sociales, éducatives, de logement ou de culture. Les processus antérieurs de décentralisation avaient coïncidé avec une intensification de la déconcentration des services de l'État, c'est-à-dire des ressources concrètes pour assurer une régulation politique du changement territorial (Epstein 2004). Les dispositifs actuels s'en distinguent. Au lieu d'un accompagnement de l'une par l'autre, se produit un transfert de l'une vers l'autre. La fin de la déconcentration signale ainsi le crépuscule d'un cycle français, assez original en Europe. Peut-on en déduire l'émergence d'un nouvel ordre territorial, fondé sur une nouvelle économie des relations entre échelles ? Selon Renaud Esptein, celle-ci s'énoncerait ainsi : l'État, dépossédé des ressources d'une régulation substantielle des enjeux territoriaux, se replierait sur un gouvernement à distance, fondé sur le pilotage réglementaire, l'encadrement normatif et la contractualisation. D'une certaine manière, le gouvernement central des territoires épouserait la logique du marché, fondant ses interventions sur la mise en compétition des pouvoirs locaux entre eux, en s'émancipant d'une dépendance territoriale désormais politiquement ingérable. Les élites locales, professionnalisées et dotées qu'elles sont de techniques et de personnels qui étaient auparavant l'apanage de la haute et centrale administration, s'intégreraient dans cette nouvelle verticalité. On voit émerger, dans cette théorie du gouvernement à distance, I'hypothèse d'une gouvernance dépolitisée des territoires qui mérite d'être soumise à critique (Jobert 2003). Loin d'être à l'abri de logiques politiques, cette évolution témoigne au contraire d'une inversion de tendance politique.

À l'idée communément admise d'un État maître de ses politiques publiques devant les négocier avec la politique incarnée par les pouvoirs locaux s'oppose aujourd'hui une vision beaucoup plus politisée de l'Etat territorial, quand les pouvoirs locaux font face, avec leurs nouvelles attributions, à des logiques croissantes de gestion de politiques publiques (Borraz \& Négrier 2007). Cette inversion revient sur la relative étanchéité que la $V^{e ̀ m e}$ République naissante avait développée à l'égard des intérêts politiques locaux. Elle rappelle une structure de l'influence directe du politique dans les enjeux territoriaux qui avait été caractéristique des III ${ }^{\text {ème }}$ et IV ${ }^{\text {ème }}$ Républiques (Le Lidec 2001). 
Aussi la croissance de l'interdépendance entre l'État et les collectivités territoriales donne-t-elle un visage assez différent de celui qui pouvait encore être décrit dans les années 1990. La repolitisation de l'État sur les politiques territoriales s'opère au travers de la pénétration croissante d'enjeux propres aux conditions partisanes et localisées de mise en œuvre. On a pu l'observer dans celle de la Loi Chevènement, à titre d'illustration (Négrier 2007). Initialement, la réforme pose une injonction à une intercommunalité centrée sur des politiques publiques urbaines (logement, développement économique, réseaux urbains, etc) en désignant des territoires élargis, au nom d'une conception volontariste de la solidarité spatiale. Mais, au cours de la mise en œuvre, le gouvernement s'avère incapable de contenir les intérêts d'une partie des élus favorables à la remise en cause des principes de solidarité affichés. Il consent à deux amendements sénatoriaux qui vident le volontarisme de substance, tout en redonnant un pouvoir d'arbitrage au cabinet du Premier ministre. Le résultat est la pénétration, au sein des politiques gouvernementales, de logiques politiques localisées. L'analyse du profil des communes autorisées à sortir d'un regroupement intercommunal, et donc à faire sécession, est très éclairante sur ce point (Jouve \& Négrier 2008). Réciproquement, une dépolitisation des collectivités territoriales s'opère au travers de la professionnalisation croissante dans la mise en œuvre de leurs attributions. Naturellement, cela ne signifie nullement que l'État ne met plus en œuvre de politiques publiques territoriales, ni que les pouvoirs locaux ne font plus de politique. Mais le jeu combiné de ces deux tendances (politisation de l'action de l'État et dépolitisation du pouvoir local) conduit à penser autrement l'interdépendance entre niveaux, mais aussi le rôle des idées dans l'action publique territoriale.

\subsection{Les idées territoriales en action}

La dimension idéologique des politiques territoriales repose sur une hypothèse de fond et suppose d'avoir résolu quelques difficultés d'interprétation. L'hypothèse est que le changement de centre de gravité des politiques publiques implique, au-delà des transferts d'attribution, des contraintes nouvelles de production, locale, de référentiels de politique publique. Le partage des valeurs, largement diffusé à l'échelle internationale, n'a pas pour conséquence l'identification de normes consensuelles pour autant; et ces normes ne donnent pas lieu à l'émergence d'algorithmes identiques. Ils sont au contraire discutables et discutés, de façon différente, au sein de configurations territoriales particulières.

Cette démonstration ne peut se passer d'un exemple, que nous allons prendre après quelques précisions utiles. La première est que cette nouvelle dimension idéologique ne signifie nullement que les territoires disposent désormais d'une capacité unilatérale de production de leur propre « monde » politique. C'est au contraire dans le contexte multi-scalaire que se produisent ces idées. Le changement réside dans la perte d'influence, sur cette production, d'acteurs qui disposaient jadis d'une influence déterminante. Sur la question urbaine, par exemple, on se réfère aux professionnels d'État. L'expertise est désormais plus diffuse, éclatée entre des bureaucraties publiques locales, des cercles d'experts provenant de l'économie mixte, de la consultance et des organismes internationaux (Cadiou 2005). La pluralité de ce milieu n'empêche pas l'émergence de discours dominants, qui donnent lieu à une 
certaine standardisation de l'offre d'idée. On en voit une illustration claire dans le développement des projets urbains (Pinson 2005).

Mais l'orthodoxie à laquelle cette standardisation pourrait conduire reste relative. Elle est d'une part caractérisée par d'importantes variations, liées à la nécessité (concurrentielle) d'innovation dans la production d'idées, sans que l'on puisse facilement démontrer la profonde unité de valeurs qui les alignerait entre elles. Un terrain urbain est, aujourd'hui, marqué par la succession de telles idées en action. Elles se repèrent tout autant dans les formes urbaines successives qui découlent de l'inspiration des urbanistes, architectes et autres aménageurs, que dans les principes qui fondent telle ou telle politique urbaine.

D'autre part, comme on va le voir plus loin par l'exemple, ces idées sont soumises à d'importantes recompositions au cours de leur mise en œuvre.

La seconde précision concerne les formes de politisation-dépolitisation des enjeux territoriaux. Alors que nous posons l'existence d'idées territoriales en action, nous assistons parallèlement au déclin de visions qui avaient constitué, au cours du XXème siècle, la trame de certaines politiques locales. Il en est ainsi du socialisme municipal, fort vecteur d'identification partisane par les politiques publiques, qui a subi, au sein du Parti socialiste, un déclin progressif (Lefebvre 2006). On pourrait se référer à deux «marqueurs », parmi d'autres, de politiques municipales de gauche, pour observer le déclin de leur capacité d'adhésion. La première est l'action culturelle, désormais presque impossible à repérer à gauche tant elle a été assumée, voire revendiquée par des équipes de droite (Négrier 2003). La seconde est constituée par les politiques de logement social, où les défaillances de mairies de gauche voisinent désormais avec la réticence plus traditionnelle de la droite dans ce domaine (Maurin 2003 ; Donzelot 2006). D'une certaine manière, c'est la fin des idéaux qui rend d'autant plus intéressante l'analyse des idées dans l'action territoriale.

Prenons, pour illustrer ce que nous venons de proposer, l'exemple de la réforme institutionnelle du gouvernement urbain. S'il s'agit d'un thème permanent des politiques territoriales, il a connu une importance croissante au fur et à mesure que l'urbanisation gagnait les périphéries des villes et métropoles. Très tôt, cette question a suscité, en Amérique du Nord, une controverse sur la manière d'y répondre. Deux idées, diamétralement opposées, ont structuré le débat.

La première, que nous nommerons réforme, proposait de répondre à l'urbanisation par la création de nouvelles institutions, afin qu'elles correspondent, par leur périmètre et par leurs compétences, à la nouvelle échelle des problèmes urbains (Woods 1958).

La seconde, qui s'inspire de l'école du public choice, prétend au contraire que la fragmentation institutionnelle, économe en bureaucratie, peut laisser le champ libre à une concurrence bénéfique entre communes. Celle-ci rend possible une amélioration générale du gouvernement local, sous la pression des habitants qui, de ce fait, feraient jouer la compétition (fiscale, d'offre de services) entre communes (Ostrom, Tiebout \& Warren 1961).

Le sort de cette controverse est intéressant, autant en Amérique du Nord qu'en France. Dans le premier cas, il apparaît nettement que le la réforme est logiquement portée par les forces de gauche, et consacrée par les chercheurs comme le courant le plus à même de répondre aux problèmes engendrés par la fragmentation 
institutionnelle (Lowery 2001, Ghorra-Gobin 2004). Il apparaît cependant que cette tendance se heurte à de nombreux obstacles politiques, et peine à démontrer ses bienfaits au concret. De plus, alors que la structure gauche-droite, au plan des idées, est assez évidente, on assiste à la mise en œuvre de projets inspirés de la réforme par des coalitions conservatrices, tandis que certaines, à gauche, plaident au contraire pour un laissez-faire digne du modèle « néo-libéral » (Keil 2000).

En Europe, où les États ont une part plus active dans la mise en œuvre de telles recompositions, de semblables difficultés d'imputation (à la gauche, à la droite) et de mise en œuvre localisée sont évidentes. Dans l'articulation entre échelles, on constate une tendance fréquente, de la part des États, à freiner l'institutionnalisation des grandes métropoles (Londres, Barcelone, Athènes); celles où, précisément, l'enjeu est le plus vif. L'idée réformiste, telle qu'elle se donne à voir dans les discours gouvernementaux, se délite au fur et à mesure de sa négociation, puis de sa mise en œuvre. Si la norme reste inchangée, les algorithmes intègrent, par compromis successifs, des ingrédients qui empruntent à chacun des idéaux : réforme/public choice.

La leçon que l'on peut tirer de ce constat ne doit pas être d'abandonner toute lecture idéelle de ces politiques publiques. Elle doit au contraire déplacer le niveau de l'interrogation, à l'échelle où ces idées se construisent au concret, c'est-à-dire au niveau urbain et métropolitain lui-même. Loin de n'être que le lieu où les idées s'éprouvent, après avoir été conçue ailleurs, les configurations territoriales sont le lieu de leur fabrique. Celle-ci se nourrit certes de multiples sources, parmi lesquelles les professionnels d'État demeurent en position d'influence, même s'ils sont divisés entre eux, en fonction notamment de leur propre perception de la question territoriale. Dans un contexte de changement institutionnel, l'homogénéité de structure, comme la forme «communauté d'agglomération » en France, ne doit pas tromper sur la forte variation de substance qu'elle recouvre au concret. Cette variation touche à tous les aspects de la politique territoriale: mode de représentation des communes dans l'institution (Le Saout et Madoré 2004) ; transfert plus ou moins effectif de telle ou telle politique publique (Négrier 2005); modalité de redistribution des ressources fiscales (Rousseau 2004), etc.

Ce sont donc les questions qui concernent les enjeux démocratiques de représentation et d'égalité, de solidarité ou de sens des politiques publiques (culturelles, sociales, de logement, etc...) qui sont en jeu. Dans ces domaines, le territoire a cessé d'être « hétéronome » pour être une sphère où se croisent les intérêts politiques, les logiques sectorielles, les professionnels de la connaissance et de l'expertise.

\section{Conclusion}

La justification d'une analyse idéelle des politiques territoriales est donc double. D'une part, elle traite d'un ensemble qui, matériellement, a pris une importance croissante à la mesure des différentes formes de dévolution, décentralisation ou fédéralisation des États. D'autre part, elle concerne la substance même des politiques publiques, et notamment celles qui touchent aux enjeux concrets d'intégration. Du même coup, la spécificité du «territoire » comme objet est de moins en moins évidente. Ce n'est pas par hasard si des notions telles que le référentiel, qui 
appartenaient au registre des politiques sectorielles, nationales le plus souvent, sont aujourd'hui utilisées pour des objets d'étude localisés. Naturellement, cette acclimatation suppose une certaine adaptation.

Sur les aspects territoriaux par lesquels Bruno Jobert a fait ses premières armes scientifiques, l'enjeu majeur était de révéler les contradictions entre les différents rôles de l'État, pour démystifier la théorie de sa neutralité (Jobert 1977b : 248). La recherche de la cohérence dans l'action publique passait pour un impératif politique, que le sociologue avait pour mission de déconstruire. L'« incohérence nécessaire » de l'État était une manière originale et paradoxale de valoriser le désordre qui se tramait sous le discours de l'action bien ordonnée. Cette fonction de dévoilement doit cependant tenir compte du fait que la plasticité, la flexibilité voire les contradictions de l'État sont aujourd'hui beaucoup plus évidentes que cachées. En attestent le flou entretenu sur les cohérences idéologiques de tel projet gouvernemental, mais aussi la multi-level politics que génère une action publique aux échelons croissants et enchevêtrés. Le politique semble même parfois doté moins d'une fonction d'assemblage que de mise en scène de sa propre diversité. On peut se demander aujourd'hui s'il ne s'agit pas aussi, voire même plutôt de rechercher les régularités éventuelles qui se cachent derrière les contradictions manifestes de l'État contemporain en action. De telles régularités participent de formes politiques, d'intérêts sociaux ou économiques qui sont moins donnés que construits. Pour les repérer, si la prise en compte des idées s'avère plus que jamais nécessaire, elle doit élargir ses terrains d'étude à la constitution idéelle des territoires. 


\section{Références citées}

Abélès, M. (1989) : Jours tranquilles en 1989, Paris: Odile Jacob.

Allies, P. (1980): L'invention du territoire, Grenoble: Presses Universitaires de Grenoble.

Amin, S. (1973), Le développement inégal. Essai sur les formations sociales du capitalisme périphérique, Paris : Éditions de Minuit

Arnaud, L, Le Bart, Ch. et Pasquier R. (2005), Déplacements idéologiques et action publique. Le laboratoire des politiques territoriales", Sciences de la Société n ${ }^{\circ} 65$, pp.3-7

Benko, G. et Lipietz A. (1992) Les régions qui gagnent : districts et réseaux : les nouveaux paradigmes de la géographie économique, Paris : PUF.

Borraz, O. \& Négrier, E. (2007), “The end of French Mayors ?”, in J. Garrard (Ed), Heads of the Local State in Past and Present, London : Palgrave (forthcoming)

Borraz, O. (1998) Gouverner une ville. Besançon,1959-1989, Rennes: Presses Universitaires de Rennes.

Briquet, J.-L. (1997), La tradition en mouvement. Clientélisme et politique en Corse, Paris : Belin.

Cadiou, S. (2005), «Vers une action urbaine «moderniste»: les effets du discours des experts savants », Sciences de la Société $n^{\circ} 65$, pp. 9-27

Castells, M. \& Godard, F. (1974), Monopolville. L'entreprise, l'État, l'urbain, Paris : Mouton Ceri, P., 1981, : "Le condizioni dello scambio politico" Quaderni di Sociologia, IV, pp.640663.

Donzelot, J. (2006), Quand la ville se défait, Paris : Le Seuil

Dulong, R. (1978) Les régions, l'Etat et la société civile, PUF Politiques Paris

Dumons, Bruno ; Pollet, Gilles et Saunier, Pierre-Yves (1997), Les élites municipales sous la IIIème République. Des villes du Sud-Est de la France, Paris : CNRS-Editions

Epstein, R. (2004) «Après la territorialisation, la différenciation territoriale ? », Pouvoirs Locaux, $\mathrm{n}^{\circ} 63, \mathrm{IV}$

Faure, A. ( 1998, dir.) Territoires et subsidiarité L'Harmattan Logiques Politiques, Paris Garraud, Ph. (1983)«Le recrutement des maires en milieu urbain : esquisse de typologie », Pouvoirs, n²4, p.29-44 
Gaudin, J.-P. (2002) Pourquoi la gouvernance?, Paris : Presses de Science-Po, coll. La bibliothèque du citoyen.

Ghorra-Gobin, C. «L'étalement de la ville américaine. Quelles réponses politiques ?», Esprit n³-4, 2004, pp.145-159

Grémion, P. (1976), Le pouvoir périphérique. Bureaucrates et notables dans le système politique français, Paris: Ed. du Seuil.

Hall, P. (1993), « Policy paradigm, social learning and the State », Comparative politics, vol. $25 \mathrm{n}^{\circ} 3$, pp. $275-296$.

Hall, P.A. and Taylor, R.C.R. (1996) 'Political science and the three new institutionalisms' Political studies, XLIV: 936-957

Harding, A. (1994), "Urban Regimes and Growth Machines. Towards a Cross-National Research Agenda", Urban Affairs Quarterly, vol. 29, no 3, 1994, p. 356-382

Jeffery, Ch. (1997, dir.), The Regional Dimension of the European Union. Towards a third level in Europe? London, Frank Cass

Jessop B. (1997), « Capitalism and its Future : Remarks on Regulation, Government and Governance », Review of International Political Economy, Vol. 4, n³, pp. 561-581

John, P. (2001), Local Governance in Western Europe, London: Sage.

Jobert, B. (1977a), "La théorie des villes comme mode de traitement de la difféence sociale", Informations sur les Sciences Sociales n¹6 (6), pp. 731-761

Jobert, B. (1977b), "Bureaucraties sociales et planification locale", Annuaire de l'Aménagement du territoire, Tome VIII, Grenoble : PUG, pp. 237-250

Jobert B. et Sellier, M. (1977), "Les grandes villes : autonomie locale et innovation politique", Revue Française de Science Politique vol.27, n², pp.205-227

Jobert, B., 2003, : «Le mythe de la gouvernance dépolitisée », in Pierre Favre, Jack Hayward er Yves Schemeil, dir., Etre gouverné. Etudes en l'honneur de Jean Leca, Paris : Presses de Science Po, pp.273-285.

Jouve, B. et Lefèvre, Ch. (1999), 'De la gouvernance urbaine au gouvernement des villes? Permanence et recomposition des cadres de l'action publique en Europe', Revue Française de Science Politique, Vol. 49 n6, pp. 835-853.

Jouve, B. et Lefèvre, Ch. (1999), Villes, Métropoles. Les nouveaux territoires du politique, Paris: Economica.

Jouve, B. et Négrier, E. (2008), «La sécession métropolitaine. Analyse comparée francoaméricaine », in Michel Camau \& Gilles Massardier (dir.), Les régimes politiques revisités, Paris : Khartala, coll. Science politique comparative, 2007

Keating, M., Loughlin J. (1996, dir.), The Political Economy of Regionalism, London : Frank Cass

Keil R., 2000, "Governance restructuring in Los Angeles and Toronto: Amalgation or secession ?", International Journal of Urban and Regional Research, 24, 4, pp. 758-781.

Lefebvre, R. (2006), «Qu'est le socialisme municipal devenu? », dans L. Arnaud, Ch. Le Bart et R. Pasquier, Idéologies et action publique territoriale, Rennes : Presses Universitaires de Rennes, pp. 51-80

Le Galès, P. \& Lequesne, Ch. (1997, dir): "Les paradoxes des régions en Europe" Paris : La Découverte 
Le Galès, P. (1997), 'Quels intérêts privés pour les villes européennes?' in A. Bagnasco et P. Le Galès, eds., Villes en Europe, Paris: La Découverte, pp. 231-254.

Le Galès, P. (2002) Le retour des villes européennes, Paris : Presses de Science Po.

Le Lidec, P. (2001), Les maires dans la République. L'association des maires de France, élément constitutif des régimes politiques français depuis 1907, Thèse Université de Paris 1 Panthéon-Sorbonne

Lefèbvre, H. (1973), Espace et politique, Paris : Anthropos (nouvelle édition, 2001)

Lenclud, G., "De bas en haut, de haut en bas, le système des clans en Corse", Etudes Rurales, $\mathrm{n}^{\circ} 101-102,1986: 137-173$

Leresche, J.-Ph. (2001) : "Gouvernance et coordination des politiques publiques", in J.P. Leresche, ed., Gouvernance locale, cooperation et légitimité. Le cas suisse dans une perspective comparée, Paris : Pédone, pp.29-65.

Le Saout, R. et Madoré, F. (2004) Les effets de l'intercommunalité, Rennes: Presses Universitaires de Rennes

Lojkine, J. (1972), La politique urbaine dans la région parisienne, Paris, Mouton

Lorrain, D. (2000) : «Gouverner les villes. Questions pour un agenda de recherche», Pôle Sud, 13, pp.27-40.

Lowery, D. «Metropolitan Governance Structures from a Neoprogressive Perspective », Swiss Political Science Revue, n7-3, 2001, pp. 130-136

Mabileau, A. (dir. 1972), Les facteurs locaux de la vie politique nationale, Paris : Pedone

Marks G. (1996), "European integration from the 1980's : state-centric v. multi-level governance.", in Journal of Common Market Studies, vol.34, n³, pp. 341-378

Maurin, E. (2004), Le ghetto français. Enquête sur le séparatisme social, Paris: Seuil, La République des Idées.

Négrier E. \& Jouve, B. (1998, dir.) Que gouvernent les régions d'Europe ?, Paris: L'Harmattan, Logiques Politiques

Négrier, E. (2003), «Politiques culturelles territoriales: dernier inventaire avant décentralisation? » L'Annuaire des collectivités locales - GRALE, Paris : CNRS Editions, pp. 47-71.

Négrier E. (2007), «Penser les changements d'échelle territoriale. Institution, dynamiques sociales et politiques métropolitaines » in A.Faure, Ph. Leresche, P.Muller \& S.Nahrath (dir.), L'action publique à l'épreuve des changements d'échelle, (à paraître)

Négrier, E. (1998), «."Échange politique territorialisé et intégration européenne" in R.Balme, A.Faure et A.Mabileau (dir.), Les nouvelles politiques locales. Dynamiques de l'action publique, Paris : Presses de Science Po, pp. 111-134

Ostrom V, Tiebout C \& Warren R. (1961) : « The organisation of Government in Metropolitan Areas : a Theoretical Inquiry », American Political Science Review $\mathrm{n}^{\circ} 55$, pp.831-842.

Pinson, G. (2005), « l'idéologie des projets urbains », Sciences de la Société nº5, pp.29-51

Pizzorno, A., 1977, : "Scambio politico e identità collettiva nel conflitto di classe" in C.Crouch et A.Pizzorno, eds, Conflitti in Europa, Milan : Etas Libris, pp. 407-433

Pourcher Y. (1995) Les maîtres de granit. Les notables de Lozère du XVIII ème siècle à nos jours, Paris : Plon 
Ritaine, E. (1989) : "La modernité localisée ? Leçons italiennes sur le développement regional” Revue Française de Science Politique Vol.39, n², pp.154-178.

Rousseau, M.P. (2004), La nouvelle architecture fiscale locale et les enjeux d'intégration et de développement économique des territoires, Rapport EIL, Université Paris XII, pour le Ministère de l'Intérieur

Sawicki F. (1997) Les réseaux du parti socialiste. Sociologie d'un milieu partisan, Paris : Belin, collection Socio-histoires

Sfez, L. (1977, dir.), L'objet local, Paris : UGE

Subirats, J. \& Gallego, R. (2002, dir.), Veinte años de autonomias en España. Leyes, políticas públicas, instituciones y opinion pública, Madrid: CIS.

Trigilia, C. (1986), Grandi partiti e piccole imprese. Comunisti e democristiani nelle regioni a economia diffusa, Bologne : Il Mulino

Wood, R. (1958) : «The New Metropolis : green belts, grass roots or Gargantua » American Political Science Review, vol 52, pp.108-122. 